\title{
BMJ Open Identifying a retrospective cohort of adolescents with chronic health conditions from a paediatric hospital prior to transfer to adult care: the Calgary Transition Cohort
}

\author{
Kyleigh Schraeder, ${ }^{\circ 1}$ Alberto Nettel-Aguirre, ${ }^{2}$ Andrew Mackie, ${ }^{3}$ Olesya Barrett, ${ }^{4}$ \\ David W Johnson, ${ }^{1,5}$ Allan Ronald Ryan, ${ }^{4}$ Gina Dimitropoulos, ${ }^{6}$ Susan Samuel ${ }^{1}$
}

\begin{abstract}
To cite: Schraeder K, NettelAguirre A, Mackie A, et al. Identifying a retrospective cohort of adolescents with chronic health conditions from a paediatric hospital prior to transfer to adult care: the Calgary Transition Cohort. BMJ Open 2019;9:e027045. doi:10.1136/ bmjopen-2018-027045
\end{abstract}

- Prepublication history and additional material for this paper are available online. To view these files, please visit the journal online (http://dx.doi org/10.1136/bmjopen-2018027045).

Received 2 October 2018 Revised 26 February 2019 Accepted 28 February 2019

Check for updates

(c) Author(s) (or their employer(s)) 2019. Re-use permitted under CC BY-NC. No commercial re-use. See rights and permissions. Published by BMJ.

For numbered affiliations see end of article.

Correspondence to

Dr Kyleigh Schraeder;

kyleigh.schraeder@ucalgary.ca

\section{ABSTRACT}

Purpose The Calgary Transition Cohort was created to examine health service utilisation by adolescents affected by chronic health conditions seen in a tertiary paediatric hospital in the province of Alberta, Canada. The cohort includes adolescents who received care before the implementation of a hospital-wide intervention to improve transitions to adult care.

Participants Using hospital records, a stepwise methodology involving a series of algorithms based on adolescents' visit frequency to a hospital ambulatory chronic care clinic (CCC) was used to identify the cohort. A visit frequency of $\geq 4$ visits in any 24-month window, during the ages of 12-17 years old, was used to identify eligible adolescents, as agreed on by key stakeholders and chronic disease clinical providers, and reflects the usual practice at the hospital for routine care of children with chronic disease.

Findings to date Adolescents with $\geq 4$ visits to the same CCC in any 2-year period $(n=1344)$ with a median of 8.7 years of follow-up data collected (range 1.4-9.1). The median age at study entry was 14 years (range 12-17) and 22 years (range 14-24) at study exit. The cohort was linked (97\% successful match proportion) to their population-level health records that allowed for examination of occurrence of chronic disease codes in health utilisation encounters (ie, physician claims, hospital admissions and emergency room visits). At least one encounter with a chronic disease code (International Classification of Diseases, 9th/10th Revisions) was observed during the entire study window in $87.9 \%$ of the cohort.

Future plans The Calgary Transition Cohort will be used to address existing knowledge gaps about health service utilisation by adolescents, seen at a tertiary care hospital, affected by a broad group of chronic health conditions. These adolescents will require transition to adult-oriented care. Longitudinal analysis of health service use patterns over a 9-year window (2008-2016) will be conducted.

\section{BACKGROUND}

Transitioning to adult care presents a major challenge for young adults with chronic health conditions. Approximately 15\%-20\%

\section{Strengths and limitations of this study}

Novel approach to identifying a retrospective cohort of adolescents with chronic physical health conditions within a paediatric tertiary care hospital in Alberta, Canada.

- Provides a description of healthcare service utilisation over a 9-year study window.

- Limited by internal hospital record data that do not require mandatory reporting.

- Limited by lack of information on disease type and mental healthcare service use by adolescents in the cohort.

of adolescents in North America live with a chronic health condition (eg, diabetes, cystic fibrosis), defined as a condition that lasts at least 3 months, is not (yet) curable, affects a child's normal activities and requires ongoing care. ${ }^{1}$ Most $(>90 \%)$ of these individuals will require transfer from paediatric to adult care. ${ }^{1}$ After the age of transfer (ie, typically 18 years old in most jurisdictions), increased visits to the emergency department (ED) are frequently reported, which is costly for adolescents and families, and for healthcare systems. ${ }^{2}$ For some adolescents, ED visits may be due to gaps in routine ambulatory care, or primary care, or a lack of developmentally appropriate services in the adult system. ${ }^{3}$ Descriptions of health service utilisation for this population have been limited to certain disease populations (eg, diabetes ${ }^{2}$, congenital heart disease ${ }^{45}$ ) and a short observation window (typically 2-years pre-transfer and post-transfer). There are key knowledge gaps regarding how adolescents living with chronic health conditions use, or do not use, healthcare services over time, specifically before and after age 18. Identifying 
a cohort of adolescents (for this paper defined as individuals between 12 years old and 17 years old) who will likely require transfer is a prerequisite for studying how to improve transitions and for efficient healthcare planning and service delivery.

Administrative data can be used to identify specific patient populations, such as adolescents who have chronic health conditions. These data are collected routinely for non-research purposes, including for patient or service management or financial reimbursement. One method of identifying patient populations using these data is to apply algorithms to disease-specific and visit-specific (eg, procedural) physician billing codes from patient encounters. In Canada, national reporting datasets (eg, claims data and hospitalisations) have professional coders who enter specific diagnostic codes, in the form of International Classification of Diseases (ICD) codes, based on hospital discharge records. ${ }^{6}$ Such coding algorithms can then be validated, for example, by comparing with patient chart records in order to detect the presence of certain conditions in a population. ${ }^{7}$ This method is often focused on disease ascertainment or identifying a patient population with a specific disease and is therefore a key source of population-based data used to estimate chronic disease prevalence. ${ }^{8}$ Validating disease-specific algorithms, however, can be costly and time-consuming. Furthermore, numerous algorithms can exist for one specific condition and can vary based on the specific setting, country or jurisdiction, complicating generalisability across settings. ${ }^{910}$

Another method of identifying specific patient populations using administrative data is using internal hospital data, ${ }^{11}$ which is unique to each health organisation and separate from national reporting data (ie, hospitalisations and ED visits). Within a hospital, healthcare professionals typically attach a hospital clinic or programme code to each patient encounter within ambulatory outpatient clinics. Using these programme or clinic codes, specific patient populations can also be identified (eg, seen by the cardiology clinic). This method is not intended to be diagnostic of a chronic condition in the individual being studied. However, it can be used to broadly define a population of adolescents who access specialized hospital services and/or programmes that provide chronic care.

The objective of the current study was to identify a cohort of adolescents living in Alberta (Canada) who attended a chronic care clinic at a tertiary paediatric hospital and who, therefore, may require transfer to adult care. This study provides health service utilisation data among adolescents currently using specialty paediatric care services (offered up to age 18 years in Alberta) for chronic health conditions. This research takes advantage of individually linked health administrative and population data available for the population of Alberta. The focus of this paper is to describe the case ascertainment methods for the cohort that we will, from now on, refer to as the Calgary Transition Cohort.

\section{COHORT DESCRIPTION}

\section{Population and setting}

The Calgary Transition Cohort was developed for the purpose of conducting a retrospective observational cohort study of a population of adolescents and young adults affected by chronic health conditions living in southern Alberta, Canada and who have received care at a tertiary paediatric hospital in Calgary, Alberta. Alberta, with a population of 4.1 million, is the fourth largest province in Canada and, like all Canadian provinces, has a universal publicly funded healthcare system that covers over $99 \%$ of the population. ${ }^{12}$ Physicians are paid for services provided to patients (hospitalised patients as well as outpatients) by the Ministry of Health. This study identified a cohort of adolescents who visited Alberta Children's Hospital, which is one of two tertiary care paediatric hospitals in the province. The Hospital provides tertiary care (outpatient, inpatient and intensive care) to all children in the Calgary Zone Health Region. This geographic region has a population of over 1.5 million residents (Census 2016), with about $18 \%$ young people (aged 10-24 years). ${ }^{13}$

\section{Methods}

Written consent from individuals was not required because data extraction and linkages were performed by Alberta Health Services (AHS) personnel (data custodian for all databases used in this study), and no identifiable data were released to the research team.

\section{Data linkage}

All Albertans are assigned a Personal Health Number (PHN) that is a unique lifetime identifier. The provincial Population Registry contains the PHNs for all individuals, and this permits linkage of an individual patient's records across all health databases and time. The Registry was used to deterministically link patient-level data to the AHS Corporate Data Repository (CDR-9), necessary for case ascertainment. The Registry was then used to confirm patient's gender and postal code. The CDR-9 is a data environment that stores a variety of data resources (eg, population cost utilisation, wait-time registry, admissions/ discharge data, deprivation index and ED data) specific to the AHS Calgary zone. The CDR-9 holds ambulatory clinic-level data unique to Alberta Children's Hospital. The Repository is the only dataset that has information on clinical programmes at the hospital.

PHNs were also used to link patients eligible for the cohort in CDR-9 to national administrative datasets. Figure 1 provides a summary of the datasets and variables used. The datasets included: National Ambulatory Care Reporting System (NACRS), Discharge Abstract Database (DAD) and the Alberta Health Practitioner claims dataset. NACRS contains data for all ED visits and day surgeries. Up to 10 diagnostic codes can be reported for each patient encounter. The DAD contains data for all hospital and intensive care unit admissions. Up to 25 diagnostic codes can be reported for each 


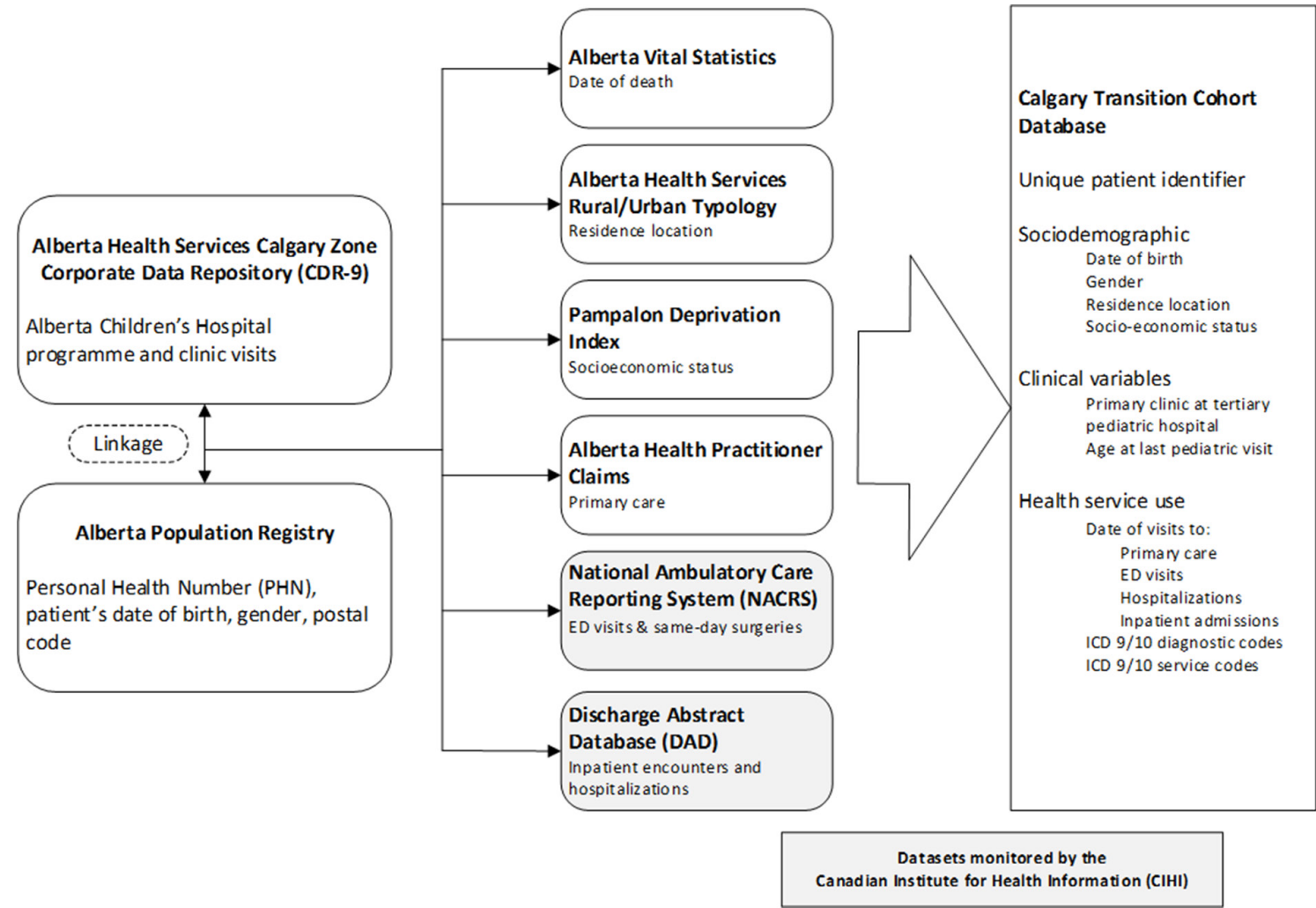

Figure 1 Datasets linked for the Calgary Transition Cohort. ED, emergency department; ICD 9/10, International Classification of Diseases, 9th/10th Revisions.

patient encounter. NACRS and DAD fall under mandatory national reporting guidelines and are separate from internal hospital records; data quality is monitored regularly by the Canadian Institute for Health Information. Finally, physician claims data contain physician billing claims. Physicians are paid for services provided to patients (hospitalised patients as well as outpatients) by the Ministry of Health, and enter their unique physician identification code to support the submission of claims. A physician claim for payment must, in addition to a billing code for the service provided, include the PHN and up to three ICD-9 diagnostic codes for the health condition(s) for which the service was provided. Claims data have face validity, and preliminary assessment of the accuracy of diagnostic recording for claims that are shadow-billed has been shown to be similar to that of claims submitted by fee-for-service physicians for certain disease groups. ${ }^{14}$

\section{Patient eligibility and cohort creation}

Patients eligible for the cohort were: (A) 12-15 years old in 2008; (B) involved with a CCC at Alberta Children's Hospital (eg, diabetes, endocrinology and nephrology; 26 clinics from CDR-9 identified after consultation with hospital clinicians and key stakeholders) and (C) who had at least four visits to the same CCC within a 2-year period, with at least 3 months between each visit, before age 18 years. In terms of CCCs, mental health programmes or clinics were not included in this study. Although some adolescents might receive mental health services within the hospital, these clinics are not uniformly captured within the CDR-9 system. Furthermore, the majority of adolescents with comorbid mental health conditions are typically referred out to community services. The age criteria was set so outcomes could be potentially observed for at least 2 years before, and after, age 18 years. The youngest patient in the cohort would therefore be 12 years old in 2008 and 20 years old at the end of the study window in 2016; the oldest patient would be 15 years old in 2008 and 23 years old at the end of observation. The accrual window for patients to enter the study thus spanned from 1 April 2008 to 31 March 2013 to allow for at least 3 years of possible observation (2013-2016) after age 18 years. The four qualifying CCC visits had to be at least 3 months apart to align with definitions of chronic health disorders in childhood. ${ }^{15}$ A flow chart of patient eligibility for the Calgary Transition Cohort is presented in figure 2. Patients were excluded if they moved out of province during the study window, as indicated in the Registry, or if they had an invalid PHN.

We acknowledge that paediatric clinic visit rates can vary based on disease type and complexity. For example, best practice guidelines for adolescents with congenital heart disease recommend only one visit per year for some types of congenital heart lesions. ${ }^{16}{ }^{17}$ Similarly, adolescents diagnosed with rare genetic disorders (eg, Turner syndrome) are typically only seen by a paediatric specialist once a year. ${ }^{18}$ To acknowledge this variation, we also examined visit frequency definitions of $<4$ visits in 24 month (ie, $\geq 2$ visits or $\geq 3$ visits to the same CCC in a 24-month window). 


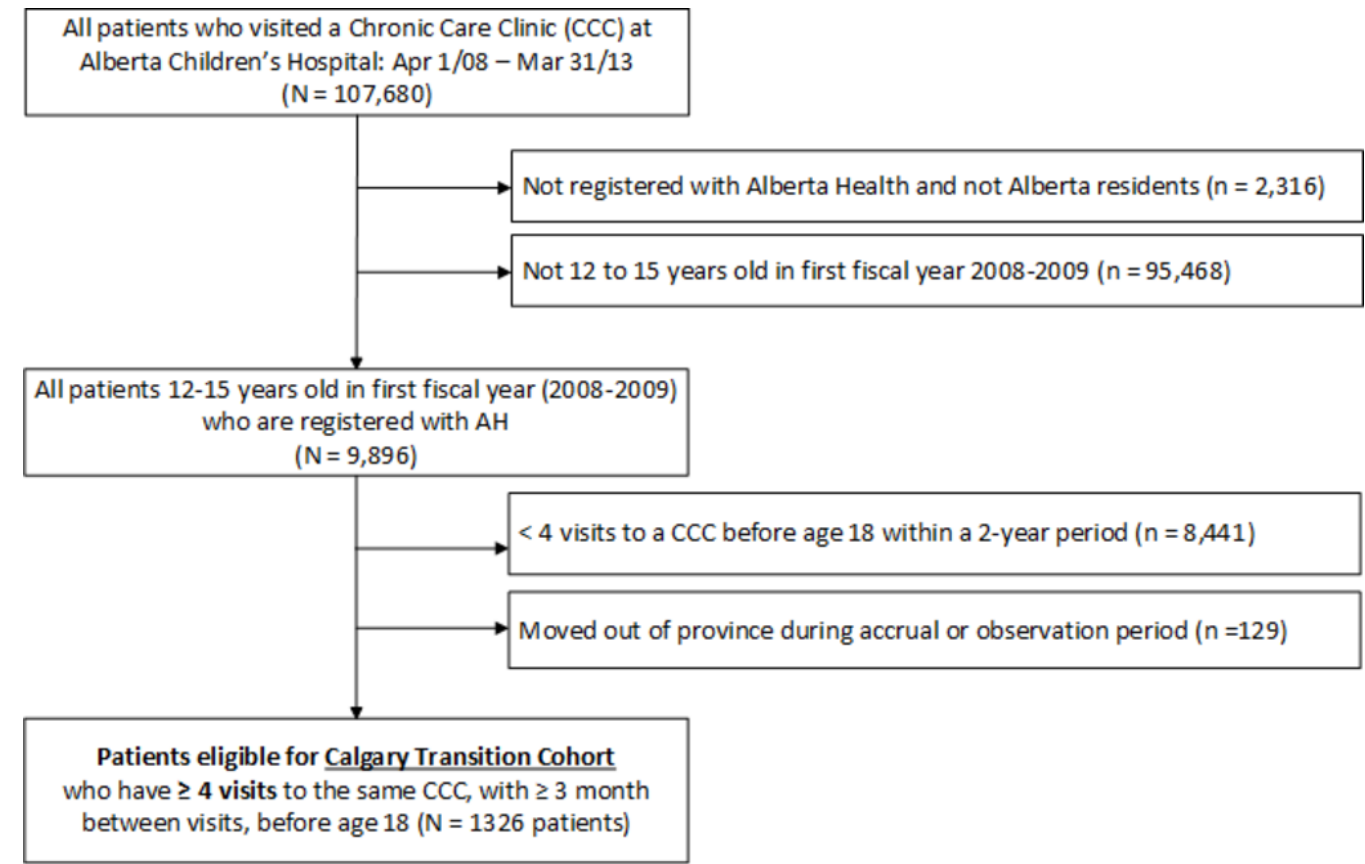

Figure 2 Cohort creation flow chart.

\section{Chronic disease screening}

A full chart validation was not feasible due to privacy regulations within the health authority and requirement for consent before examination of clinical records. A screen of the Calgary Transition Cohort for chronic disease diagnostic codes was conducted by examining the proportion of patients who had at least one ICD-9 (or 10) chronic disease code, ${ }^{6}$ as developed by Feudtner et al, for encounters recorded in administrative health datasets (ie, emergency visits, inpatient visits and hospitalisations, and physician billing claims). The original Feudtner classification was developed based on input from clinical professionals and a literature review of studies identifying children with complex and high-cost conditions. ${ }^{6}$ The codes were grouped into 10 groups based on body system or disease origin. Examples of conditions include cerebral palsy, epilepsy (not including benign childhood epilepsy [G40.0] and petit mal [G40.7]), organ transplantation and dependence on devices. For the purpose of this study, ICD 9/10 codes for other less complex chronic diseases, specifically asthma and diabetes, were also included. Our adapted chronic disease code list is listed in the (online supplementary table S1). We also applied our code list to patients who did not meet our CCC visit requirement or who had fewer visits to the same CCC in a 24-month window (ie, $<4$ visits). This allowed us to further assess our patient eligibility criterion. We hypothesised the visit rate criterion (ie, $\geq 4$ visits to the same CCC in a 24 -month window) would be more specific for capturing a patient population affected by chronic illness (ie, having at least one chronic disease code during the study window) than alternate visit ratio criteria (ie, $\geq 2$ visits or $\geq 3$ visits to the same CCC in a 24-month window).
Cohort description

Patient's sex, birth dates and postal codes were obtained from the Vital Statistics dataset. For the purposes of this study, Census 2011 postal codes were used. Patient's residence location (ie, urban or rural) was based on their postal code on entry into the cohort and the AHS RuralUrban Typology. This typology contains the rural-urban continuum areas based on the aggregation of local geographic areas. The rural-urban continuum is divided into seven distinct areas (ie, metro, urban, moderate metro influence, moderate urban influence, rural, rural remote, large rural centres and surrounding areas). The metro areas span from populations of $\geq 25000$ (urban areas) to $>500000$ (metro centre); remote and rural areas span from $<10000$ to $<25000$. The rural-urban continuum was dichotomised in this study: (1) urban (ie, metro, urban, moderate metro influence and moderate urban influence) and (2) rural (ie, rural, rural remote, large rural centres and surrounding areas).

Patient's area-level socioeconomic status was determined using the material deprivation component of the Alberta Pampalon Deprivation Index. ${ }^{19}$ This index is a small area-based composite index that uses Canadian census data at the dissemination area level to present socioeconomic disparities among the population. The material deprivation component of the PDI is most relevant and applicable in the Alberta context as the social component of the PDI is not adjusted to Alberta population's age and sex. ${ }^{19}$ The material deprivation component consists of three indicators: (1) the proportion of people aged 15 years and older with no high school diploma, (2) the population/employment ratio of people aged 15 years and older and (3) the average income of people aged 15 years and older. This index stratifies the population into 
five quintiles with the highest quintile (Q5) representing individuals in the population living in areas with most deprivation.

Patient's primary CCC at the Alberta Children's Hospital, or the clinic for which patients entered the cohort, and frequency of CCG visits during the study window was obtained from the CDR-9. National reporting datasets provided information on the patient visit rate (per person years) by age (eg, 12-<13 years old, $13-<14$ years old and $14-<15$ years old) for visits to a family physician (physician claims) and ED (NACRS).

\section{Patient and public involvement}

The issue of transition from paediatric to adult care is a key priority for patient groups across Alberta, Canada. Although patients and the public were not directly involved in the creation of this retrospective cohort, this study is part of a larger research programme on transitions in care at the Alberta Children's Hospital, which involves patient advisors. Preliminary findings from this cohort have been presented to our team and patient advisors. Further analyses will be guided by input by patients and citizens.

\section{FINDINGS TO DATE \\ Cohort characteristics}

Using the CDR-9, a total of 107680 patients visited a CCC at the Alberta Children's Hospital between 2008 and 2013. Two per cent $(n=2,316)$ were excluded due to living outside of Alberta or having an invalid PHN, resulting in a $98 \%$ match to Registry data. Of the 105 364 patients with a valid PHN, 90.6\% did not meet age-related inclusion criteria for the study (ie, were not between the ages of 12-15 at the start of the study window). The final sample consisted of 1326 patients who had at least four visits to a CCC within a 2-year period before age 18 years.

Table 1 provides a summary of the demographic and clinic-related variables for the Calgary Transition Cohort $(\mathrm{n}=1,326)$. The median age at study entry (ie, at the first of 4 qualifying CCC visits) for patients in the cohort was 14 years (range $12-17$ years), and at study exit was 22 years (range 14-24 years). The median age at last observed visit to a CCC at the paediatric hospital was 17 years old; with the highest age observed being 24 years old.

About a third of adolescents (32\%) were involved with more than one hospital CCC during the study window. Our cohort was primarily composed of patients who entered our cohort through clinics for: diabetes $(21.3 \%)$, gastroenterology (12.5\%), rheumatology (9.8\%), neurology $(8.4 \%)$, endocrinology $(6.9 \%)$, cardiology $(6.2 \%)$ and nephrology $(6 \%)$. These clinics cover approximately $70 \%$ of all patients who entered our cohort. The median duration of observation was 8.7 person years (range $=1.3-9.1$ years).
Table 1 Characteristics of the Calgary Transition Cohort $(n=1326)$

\begin{tabular}{|c|c|}
\hline Characteristics & $\begin{array}{l}\text { Proportion of Calgary } \\
\text { Transition Cohort } \\
(n=1326)\end{array}$ \\
\hline \multicolumn{2}{|l|}{ Demographic } \\
\hline \multicolumn{2}{|l|}{ Child's sex (\%) } \\
\hline Female & 50.2 \\
\hline Male & 49.8 \\
\hline Deaths during study window & 2.1 \\
\hline Average age at death & 18.9years old \\
\hline \multicolumn{2}{|l|}{ Residence location*† (\%) } \\
\hline Urban/metro & 85.4 \\
\hline Rural & 14.6 \\
\hline \multicolumn{2}{|c|}{ Pampalon Deprivation Index $\ddagger$ (\%) } \\
\hline Q1 (least deprived) & 18.6 \\
\hline Q2 & 20.8 \\
\hline Q3 & 20.1 \\
\hline Q4 & 17.9 \\
\hline Q5 (most deprived) & 18.0 \\
\hline Missing & 4.5 \\
\hline \multicolumn{2}{|l|}{ Clinical } \\
\hline \multicolumn{2}{|c|}{ Number of CCCs adolescents involved with§ (\%) } \\
\hline 1 clinic only & 67.6 \\
\hline$>1$ clinic & 32.3 \\
\hline \multicolumn{2}{|c|}{ Most frequently visited CCC during study window (\%) } \\
\hline Diabetes & 21.2 \\
\hline Gastroenterology & 13.2 \\
\hline Rheumatology & 12.1 \\
\hline Neurology & 8.6 \\
\hline Endocrinology & 7.1 \\
\hline Nephrology & 7.1 \\
\hline Neuromotor & 4.2 \\
\hline Asthma & 3.4 \\
\hline Oncology & 3.3 \\
\hline Ophthalmology & 2.8 \\
\hline Cardiology & 2.2 \\
\hline
\end{tabular}

${ }^{*}$ At study entry.

†As defined by Alberta Health Services urban and rural typology. $\ddagger$ Pampalon Deprivation Index (Pampalon et al) using 2011 census data.

§During study window.

ПClinics representing over $2 \%(n=28)$ of sample were not reported. CCC, chronic care clinic at Alberta Children's Hospital.

\section{Chronic disease screening}

All patients in the cohort were successfully linked to population-level datasets (NACRS, DAD and claims). The proportion of patients who had at least one healthcare visit during the study window that was associated with a chronic disease ICD-9/10 code was $87.9 \% \quad(n=1165)$. We also examined broader definitions of a chronic care patient, who had less than the required four visits to the 


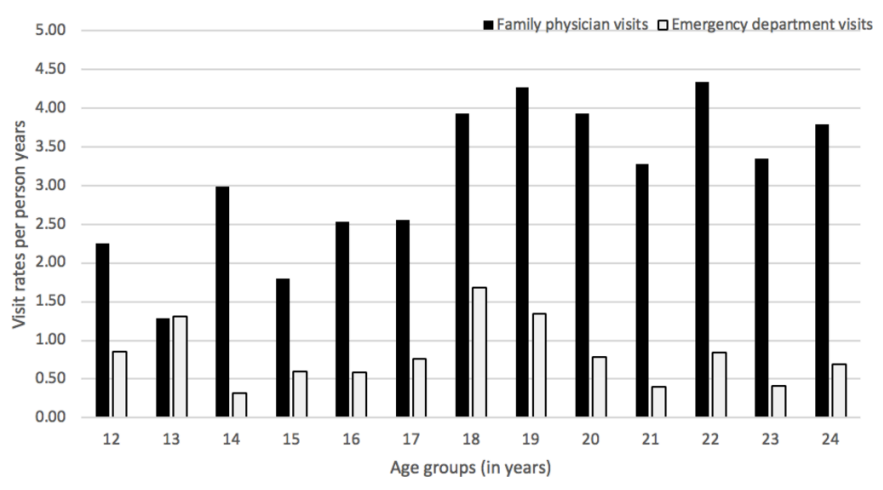

Figure 3 Comparison of age-specific visit rates, per person years, to family physicians and an emergency department in the Calgary Transition Cohort $(n=1326)$.

same CCC within a 24-month window, and applied our adapted chronic disease code list. For patients with $\geq 2$ visits to a CCC $(\mathrm{n}=4068), 76.9 \%$ had at least one chronic disease code. For patients with $\geq 3$ visits to a CCC ( $n=2213)$, $83.2 \%$ had at least one chronic disease code.

\section{Health service utilisation for Calgary Transition Cohort}

Age-specific visit rates are presented in terms of visits per person years for each type of service utilisation (figure 3 ). As shown in figure 3 , at age 15 years, the rate of visiting a family doctor in that given age period was 1.8 visits; after age 18 years, the rate of visiting a family doctor ranged between 3.9 and 4.3 visits per year. Based on the patient's most frequently visited CCC during the study window, patients who had the highest overall rates of primary care use (ie, visits to family physician) over the 8-year study window were those in the cardiology clinic ( $\mathrm{n}=29 ; 4.9$ visits), neurology clinic $(\mathrm{n}=114 ; 4.3$ visits $)$ and gastroenterology clinic $(\mathrm{n}=175$; 4.1 visits). Similarly, patients in the ear-nose-throat clinic ( $\mathrm{n}=35 ; 1.4$ visits $)$, neurosurgery clinic ( $\mathrm{n}=111 ; 1.1$ visits) and cardiology clinic ( $\mathrm{n}=83 ; 1.1$ visits) had the highest overall rate of ED use, per person years, over the study window.

\section{DISCUSSION}

The current study described methods for the creation of the Calgary Transition Cohort, a cohort of adolescents living in Alberta who have attended a CCC (eg, diabetes) at a tertiary care pediatric hospital in Calgary, Alberta, Canada. Patients who entered the cohort were between 12 years and 17 years of age and had at least four visits to a CCC within a 2-year period. To our knowledge, this cohort represents the first of its kind in Alberta and is an important starting point for understanding patterns of service utilisation by adolescents involved with tertiary chronic care services.

We found that $87.9 \%$ of patients in the Calgary Transition Cohort had at least one healthcare visit (ie, outpatient, inpatient or emergent care) in the province with a chronic disease code (claim), using a modified version of Feudtner's ICD-9/10 coding system, representing 12 main areas of chronic conditions (eg, respiratory, renal and urological). ${ }^{6}$ There are several reasons why $14 \%$ of patients in our cohort who attended a CCC at the hospital did not have a healthcare visit associated with a chronic disease code. First, some patients might have had four visits to a CCC at the hospital (ie, our inclusion criteria) but did not actually have a chronic condition or have a confirmed diagnosis. Furthermore, some paediatric subspecialists in hospital are salaried (not 'fee-for-service') and use a 'shadow-billing' process, which involves submitting information for services provided as if they were submitting a fee-for-service claim even though physician payment is not directly linked to the services reported. Patients who received services by a physician who used 'shadow-billing' may not have all applicable disease codes captured in the administrative datasets. Finally, not all hospital clinics or programmes (eg, eating disorders programme and cleft palate clinic) had a corresponding ICD-9 or ICD-10 code in the adapted Feudtner's list; however, these clinics represented $<1 \%$ of our cohort.

There are two main strengths to the novel approach we used to create the Calgary Transition Cohort. First, we used internal hospital administrative data to identify our patient cohort, which was relatively inexpensive to access and could be deterministically linked to other population-level data sources. This allowed us to examine patient service utilisation in other areas of healthcare (eg, primary care), over a 9-year window. Second, our cohort was based on a select number of specific hospital clinics and/or programmes known to provide chronic care. We identified a cohort of patients who accessed tertiary care and who may have a chronic health condition, an assumption made due to their repeated attendance at a CCC $(\geq 4$ visits in a 24-month window). When the visit eligibility criterion was changed to include patients with fewer visits to a CCC (ie, $\geq 2$ or $\geq 3$ visits), the proportion of patients who positively screened for having at least one chronic disease code during the study window decreased. Our visit criterion was therefore appropriate for capturing a cohort of adolescents likely to be affected by a chronic illness.

Our approach for cohort creation is not diagnostic for a chronic condition and did not rely on complex diagnostic coding algorithms. It is therefore possible that our cohort captured adolescents who may not yet have a clear diagnosis but who are still involved in chronic care services provided by a tertiary care paediatric hospital. It is possible that requiring more than one visit (claim) with a chronic disease ICD code may have increased specificity or adolescents in our cohort who actually have a chronic health condition. The goal of this study, however, was to understand health service utilisation by adolescents who are currently using paediatric tertiary care services.

As in all secondary health data research, our study is not without limitations. One limitation, inherent to using internal hospital data (CDR-9), was that it was not possible to determine the proportion of missing visit data. Since hospital clinic data do not fall under mandatory reporting requirements, the data quality of visit reporting is expected to vary (eg, between providers and/or clinics). 
As a result, our cohort likely represents an underestimate of all adolescents seen at a chronic care clinic. Another related limitation concerns who this cohort includes and who it does not. Our cohort represents a unique subset of individuals who have accessed tertiary-level specialty healthcare services. It does not include adolescents waitlisted for tertiary level services or who are primarily managed by providers in the community (eg, by a community paediatrician or family physician). Our cohort therefore has the potential to underestimate certain paediatric chronic health conditions (eg, asthma; only $3.4 \%$ of our sample) that are commonly managed in community primary healthcare settings. Finally, our cohort cannot provide information about patterns of health service utilisation based on specific childhood diseases or conditions. A full chart validation was not feasible due to privacy regulations, and therefore, it is not possible to conduct a gold standard validation.

We also acknowledge the limitation of focusing exclusively on adolescents who have chronic physical conditions and not including neurodevelopmental conditions (eg, attention-deficit hyperactivity disorder and autism spectrum disorders) or common adolescent mental health conditions (eg, depression and anxiety), which can also be chronic. ${ }^{20}$ A separate study, using different sources of data, would be needed to explicitly investigate health service use of adolescents who have accessed mental health services.

\section{IMPLICATIONS AND FUTURE PLANS}

This study presents preliminary data on age-specific visits rates (ie, ED and primary care) by a cohort of adolescents seen at a tertiary care paediatric hospital. Higher visit rates were observed for both ED and primary care use at age 18 years, though rates varied in the years leading up to, and after, the age of transfer. Population-based rates of ED and primary care utilisation by 'healthy' adolescents, who do not have a chronic physical health condition for example, is needed to interpret our findings, and this work is currently underway. Longitudinal analyses of the Calgary Transition Cohort will be conducted to examine patterns of health service use over a 9-year window (20082016) and to examine the association of demographic (eg, gender) and clinic variables (eg, primary hospital clinic) to distinct patterns of health service use. This cohort may be linked to social services administrative data to gain a better understanding of other types of concurrent service use in other sectors of care (eg, education and juvenile justice). This information is needed to support the integration of specialty services with other types of services to improve continuity of care for adolescents with chronic physical health conditions.

\footnotetext{
Author affiliations

${ }^{1}$ Department of Pediatrics, University of Calgary, Calgary, Alberta, Canada ${ }^{2}$ Cumming School of Medicine, University of Calgary, Calgary, Alberta, Canada ${ }^{3}$ Department of Pediatrics, University of Alberta, Edmonton, Alberta, Canada ${ }^{4}$ Alberta Health Services, Calgary, Alberta, Canada
}

${ }^{5}$ Department of Emergency Medicine, Alberta Children's Hospital, Calgary, Alberta, Canada

${ }^{6}$ Faculty of Social Work, University of Calgary, Calgary, Canada

Contributors KS contributed to all major aspects of this project including the cohort creation, analysis and writing of this manuscript. AN-A contributed to the study design, provided statistical input on the cohort creation and methodology, interpretation of results and writing of this manuscript. AM is a coprincipal investigator on the grant that supported this work. He contributed to the study design, interpretation and writing of this manuscript. $\mathrm{OB}$ performed all the analyses for this work within Alberta Health Services. She contributed to the study design. DWJ and ARR contributed to the interpretation of findings and cohort creation. GD is a coprincipal investigator on the grant that supported this work. She contributed to the interpretation and writing of this manuscript. SS is the lead principal investigator on the grant that support this work. She contributed to all major aspects of this project, including the study design, interpretation and writing of this manuscript.

Funding This study was performed within the Data Integration, Measurement and Reporting (DIMR) programme of Alberta Health Services. This work was supported by a Canadian Institutes for Health Research grant (\#10020324). KS holds a Canadian Child Health Clinician-Scientist Program postdoctoral award.

Competing interests None declared.

Patient consent for publication Not required.

Ethics approval This study was approved by the University of Calgary Conjoint Health Research Ethics Board (REB 14-1286).

Provenance and peer review Not commissioned; externally peer reviewed.

Data sharing statement Health record data used to create this cohort are not available for public use. Alberta Health Services is the custodian of these data.

Open access This is an open access article distributed in accordance with the Creative Commons Attribution Non Commercial (CC BY-NC 4.0) license, which permits others to distribute, remix, adapt, build upon this work non-commercially, and license their derivative works on different terms, provided the original work is properly cited, appropriate credit is given, any changes made indicated, and the use is non-commercial. See: http://creativecommons.org/licenses/by-nc/4.0/.

\section{REFERENCES}

1. Kaufman M, Pinzon J. Adolescent Health Committee. Transition to adult care for youth with special health care needs. Paediatr Child Health 2007;12:785-8.

2. Nakhla M, Daneman D, To T, et al. Transition to adult care for youths with diabetes mellitus: findings from a Universal Health Care System. Pediatrics 2009;124:e1134-41.

3. Gray WN, Holbrook E, Morgan PJ, et al. Transition readiness skills acquisition in adolescents and young adults with inflammatory bowel disease: findings from integrating assessment into clinical practice. Inflamm Bowel Dis 2015;21:1125-31.

4. Mackie AS, Ionescu-Ittu R, Therrien J, et al. Children and adults with congenital heart disease lost to follow-up: who and when? Circulation 2009;120:302-9.

5. Gurvitz MZ, Inkelas M, Lee M, et al. Changes in hospitalization patterns among patients with congenital heart disease during the transition from adolescence to adulthood. J Am Coll Cardiol 2007;49:875-82.

6. Feudtner C, Feinstein JA, Zhong W, et al. Pediatric complex chronic conditions classification system version 2: updated for ICD-10 and complex medical technology dependence and transplantation. BMC Pediatr 2014;14:1-7.

7. Muggah E, Graves E, Bennett C, et al. Ascertainment of chronic diseases using population health data: a comparison of health administrative data and patient self-report. BMC Public Health 2013;13:16.

8. Lix LM, Yogendran MS, Shaw SY, et al. Population-based data sources for chronic disease surveillance. Chronic Dis Can 2008;29:31-8.

9. Tonelli M, Wiebe N, Fortin M, et al. Methods for identifying 30 chronic conditions: application to administrative data. BMC Med Inform Decis Mak 2015;15:31.

10. Neff JM, Sharp VL, Muldoon J, et al. Identifying and classifying children with chronic conditions using administrative data with the clinical risk group classification system. Ambul Pediatr 2002;2:71-9. 
11. Wijlaars LP, Gilbert R, Hardelid P. Chronic conditions in children and young people: learning from administrative data. Arch Dis Child 2016;101:881-5.

12. Alberta Health. Alberta Health: what is covered under the AHCIP. http://www.health.alberta.ca/AHCIP/what-is-covered.html (Published 2018).

13. Statistics Canada. Census profile, 2016 census. http://www12. statcan.gc.ca/census-recensement/2016/dp-pd/prof/details/page. $\mathrm{cfm}$ ?Lang=E\&Geo1=HR\&Code1 $=4832 \& G e 02=\mathrm{PR} \&$ Code2 $=48 \&$ Data $=$ Count\&SearchText=CalgaryZone\&SearchType=Begins\&SearchPR $=$ 01\&B1=All\&GeoLevel=PR\&GeoCode=4832\&TABID=1 (Published 2016).

14. Fathima S, Simmonds KA, Drews SJ, et al. How well do ICD-9 physician claim diagnostic codes identify confirmed pertussis cases in Alberta, Canada? A Canadian Immunization Research Network (CIRN) Study. BMC Health Serv Res 2017;17:1-7.

15. Mokkink LB, van der Lee JH, Grootenhuis MA, et al. Defining chronic diseases and health conditions in childhood $(0-18$ years of age): national consensus in the Netherlands. Eur $J$ Pediatr 2008;167:1441-7.

16. Wernovsky G, Rome JJ, Tabbutt S, et al. Guidelines for the outpatient management of complex congenital heart disease. Congenit Heart Dis 2006;1:10-26.

17. Haas NA, Schirmer KR. Guidelines for the management of congenital heart diseases in childhood and adolescence. Cardiol Young 2017;27:S1-105.

18. Frias J, Davenport M. The Committee on genetics, and the section on endocrinology. health supervision for children with turner syndrome. Pediatrics 2003;99:145-6.

19. Pampalon R, Hamel D, Gamache P, et al. A deprivation index for health planning in Canada. Chronic Dis Can 2009;29:178-91.

20. Schraeder KE, Reid GJ. Who Should Transition? Defining a Target Population of Youth with Depression and Anxiety That Will Require Adult Mental Health Care. J Behav Health Serv Res 2017;44:316-30. 